\title{
Categorization of first-year university students' interpretations of numerical linear distance-time graphs
}

\author{
Thomas Wemyss and Paul van Kampen* \\ Centre for the Advancement of Science and Mathematics Teaching and Learning \& School of Physical Sciences, \\ Dublin City University, Glasnevin, Dublin 9, Ireland \\ (Received 15 May 2012; published 15 February 2013)
}

\begin{abstract}
We have investigated the various approaches taken by first-year university students $(n \approx 550)$ when asked to determine the direction of motion, the constancy of speed, and a numerical value of the speed of an object at a point on a numerical linear distance-time graph. We investigated the prevalence of various well-known general graphing difficulties, such as graph-as-picture errors and slope-height confusion. We established that two-thirds of our students could determine the direction of motion with respect to a reference point, just under $80 \%$ could determine that the speed is constant, and just under $20 \%$ of our students could correctly determine the value of the speed; in the latter case, about half of the students divided the two coordinates. Three stable categories of correctly explaining the constancy of speed emerged from the data. We found that the reason given for determining that the speed of the object was constant did not correlate with successfully determining a value for the speed. We have established that technical difficulties such as determining the slope of any linear graph did not explain the poor performance. By comparing the answers to similar questions on water level versus time graphs, we were able to establish that context dependence and incorrect prior learning are likely to play a role. Posttest data are used to confirm the validity of the categorization and support the conclusion that being able to determine the slope of a $y, x$ graph and having a correct qualitative understanding of a distance-time graph is not sufficient to correctly determine a value for the speed.
\end{abstract}

DOI: 10.1103/PhysRevSTPER.9.010107

PACS numbers: 01.40.Fk

\section{INTRODUCTION}

In this paper we investigate the approaches taken by first-year university undergraduate students when asked questions on numerical distance-time graphs. All graphs were linear and did not go through the origin. We asked students to determine in what direction an object was moving and whether its speed was constant, and to find a numerical value for the speed at a particular instant. Thus, we asked the students to engage with a graphical representation of uniform motion, to differentiate between distance and speed, to use (implicitly or explicitly) the idea of a limit, and to use an algorithm for determining the slope of a graph. We have also investigated whether there was a context dependence to the approaches our students took.

In Sec. IA we consider some relevant findings in the literature regarding difficulties with graphing and graph interpretation. In Sec. IB we discuss some difficulties students may have in distinguishing distance and speed in a classroom setting.

\footnotetext{
*Paul.van.Kampen@dcu.ie

Published by the American Physical Society under the terms of the Creative Commons Attribution 3.0 License. Further distribution of this work must maintain attribution to the author(s) and the published article's title, journal citation, and DOI.
}

\section{A. Research on graphing}

Early research in both science and mathematics education identified and documented strategies students use when they are asked to switch between graphs, other representations, and observable events in general $[1,2]$. Student difficulties with distance-time graphs, in particular, have been the subject of a number of investigations [1-8]; see also the extensive review by McDermott and Redish [9]. We use the general categorization of student difficulties with graph interpretation given by Leinhardt et al. [2] to summarize the many findings. They distinguish between interval-point confusion, graph-as-picture error, and slope-height confusion.

(1) An example of interval-point confusion occurs when one quantity is greater than another over a certain interval, but students only discuss a single point on that interval. For example, in the context of Fig. 1(a), an interval-point confusion would occur if somebody were asked when the ball is more than $1 \mathrm{~m}$ away and replied with a single instant (say, $t=4 \mathrm{~s}$ ) when a fully correct answer would refer to an interval (typically containing the instant mentioned; in this case, between 2.6 and $5 \mathrm{~s}$ ).

(2) Students make a graph-as-picture error, e.g., when they interpret a distance-time graph as a road map or a simplified sketch of a journey. In the former case, they may represent an object first moving to the right and then moving to the left by a line on a 
A ball moves along a track. The graph at right shows the distance from the ball to a fixed point during a number of seconds.

a. Is the ball moving away from, moving towards, or not moving with respect to the fixed point? Explain.

b. Is the speed of the ball increasing, decreasing, or constant? Explain.

c. What is the speed of the ball at $t=3.0$ s? Explain.

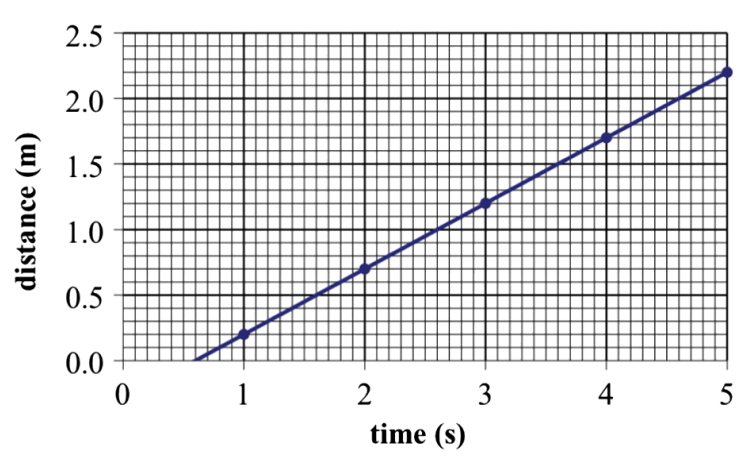

(a)
What is the slope of the line shown at right at the point indicated with the dot? Explain briefly, and show your work.

(b)

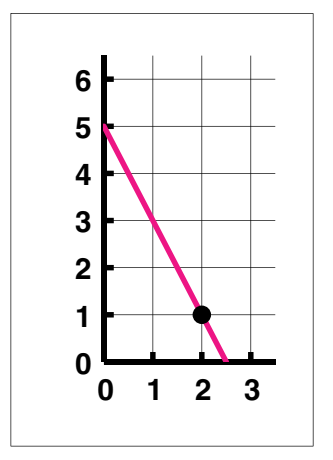

The graph at right shows the water level in a flat-bottomed swimming pool at different times.

a. Is the water flowing into the pool, out of the pool, or does water not flow at all? Explain.

b. Is the water level changing at an increasing, decreasing, or constant rate? Explain.

c. How quickly does the water level change at $t=200 \mathrm{~s}$ ? Explain.

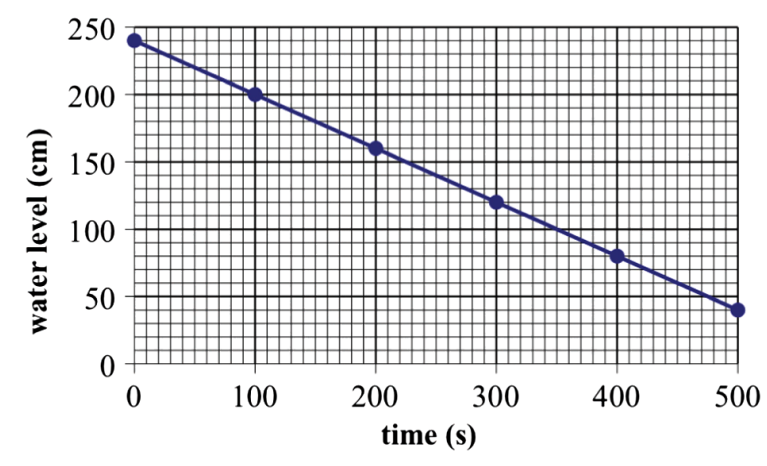

(c)

FIG. 1 (color online). Typical graphs used to investigate difficulties with determining slope from a linear graph: (a) distance-time graph; (b) y, $x$ graph; (c) water level versus time graph. On some occasions, the best-fit line was omitted from graphs like (a) and (c). This made no significant difference to the answers given.

distance-time graph that first moves to the right and then to the left (i.e., with time decreasing); in the latter case, a distance-time graph may be interpreted as a graph showing altitude versus horizontal distance. Thus, a student could interpret Fig. 1(a) as showing that the ball rolls uphill.

(3) The third category, slope-height confusion, refers to graphs where students confuse the slope of a graph with the coordinates $[4,7,8]$. Thus, students might interpret Fig. 1(a) as showing that at $t=1 \mathrm{~s}$, the speed equals $0.2 \mathrm{~m} / \mathrm{s}$ rather than the distance being $0.2 \mathrm{~m}$; or they might reason that the speed of the ball is increasing, rather than the distance.

\section{B. Slope-height confusion or speed-distance confusion?}

It is often not easy to distinguish between difficulties with graph interpretation per se, difficulties with rate of change versus value in general, or difficulties with the concepts of speed and distance. It may seem so unlikely that university science students would confuse speed and distance that one may be tempted to dismiss the possibility out of hand. We do not think that any of our students have difficulty distinguishing speed and distance in everyday life. Quite apart from whatever formal education they have had, the (unquantified) notions of distance and motion are interlinked at a very deep level: in the first month of their 
lives, humans develop their depth perception largely based on kinetic cues [10]. By the age of 11, prior to formal learning, almost all people have developed a cognitive framework in which distance and speed have a direct relationship, as do distance and duration, while duration and speed have an inverse relationship [11]. However, this is not to say that students can distinguish between the concepts of speed and distance as defined in the classroom.

We give two examples of how confusion between distance and speed may occur in the context of formal education. First, all of the most popular school science textbooks that would have been used by our student cohort define speed in words as "the distance travelled by an object in one unit of time" [12]. A literal interpretation of this definition would lead one to believe that speed is a distance. Second, students have been taught that the product of two numbers $a$ and $b$ is to be interpreted as the successive addition of $b$ terms of $a$ (or vice versa). Seen in this light, it is not unreasonable to think of the distance traveled, $d$, as a result of adding $t$ unit distances $v$ to each other.

Thompson [13] discusses the somewhat analogous case of a 10-year-old student, whose initial conceptual understanding may be summarized as follows: speed is the distance traveled in $1 \mathrm{~s}$, and time is the ratio of distance and speed. Thus, if an object travels at constant speed $v$, then $v$ is the distance traveled in $1 \mathrm{~s}$; the ratio $d / v$ gives the number of unit distances $v$ required to make up the total distance $d$; and this ratio is identified as the time taken to travel. We do not know how prevalent this kind of reasoning is, but Thompson certainly presents a compelling example of how students may be able to successfully carry out and discuss calculations involving (constant) speed, distance, and time to considerable depth, and yet not see speed as very different from distance.

\section{THEORETICAL CONSIDERATIONS}

On the theoretical side, a number of frameworks have emerged that aim to interpret the findings described above. Of late, there has been a shift away from merely identifying misconceptions (reasonably stable concepts that form part of a more or less coherent framework different from that of experts) to focusing on capabilities [14] and knowledge in pieces [15] - for example, in the form of phenomenological primitives [16] or p-prims (small pieces of knowledge assembled from experience), facets [17], or affordances, which may be defined as possible actions that are likely to be triggered, in particular, settings [18].

All capabilities theories stress the importance of context. Context generally plays an important role when students answer questions that require interpretation of graphs. Roth [19] points out that in order to answer a graphing question, students need to have both an understanding of the context and a general ability to interpret graphs. Tests by ÅbergBengtsson and Ottosson [20] and the Test of Graphing in Science [5] aimed at middle school students found a strong context-dependence of student performance on graph interpretation questions. Heckler et al. [21] recently found that students reply differently to similar questions on height and slope depending on the variables plotted on otherwise identical graphs.

By contrast, Elby has claimed that "in a visual representation, the compelling visual attribute tends to cue WYSIWYG," where WYSIWYG stands for "whatyou-see-is-what-you-get" and that a straight line graph cues constancy [22]. This suggests that at least some elements of productive graph interpretation may not depend on context after all.

\section{NUMERICAL DISTANCE-TIME GRAPHS}

In this work, numerical distance-time graphs take center stage. The research started with asking first-year university science students to determine the speed at a particular instant from a linear distance-time graph that does not go through the origin. An example is given in Fig. 1(a). We typically asked students three questions, in the same order:

(a) The direction the object is moving in;

(b) Whether the speed is increasing, decreasing, or constant;

(c) The value of the speed at a particular instant.

Minor variations of the questions exist. In some case, we labeled the reference point $P$; sometimes only data points were given but no line. Most importantly, some of the graphs have a positive slope, while others have a negative slope.

In principle, these questions may be answered correctly with only knowledge of the relevant definitions, i.e., without understanding the underlying physics. For example, to determine the speed from a distance-time graph, students must be able to calculate a value for the slope. All students have encountered the concept in mathematics, and most are familiar with the formula for the slope of a straight line,

$$
\text { slope }=\frac{y_{2}-y_{1}}{x_{2}-x_{1}} .
$$

As the slope is constant, the theory of limits allows us to ascribe this ratio to the slope at any point on the graph [23].

It seems likely that students must be able to use (1) implicitly or explicitly to extract the speed of an object at an instant from a distance-time graph. In fact, students who are able to apply a technique to determine the slope of a $y, x$ graph only need to substitute the word "speed" for "slope," and append appropriate units to obtain a correct numerical answer. A deeper understanding, e.g., recognition that because the ball travels at constant speed the instantaneous speed is equal to the average speed during any interval, is not required to "get the right answer."

Notwithstanding these considerations, we expected context to play an important role in the students' approaches to answering questions on distance-time graphs, as prior learning can be expected to play a role. Many high school and university students have been found to struggle when 
they are asked to determine the speed from a distance-time graph, especially when the graph is not a straight line through the origin. For example, Beichner [24] found that $73 \%$ of 895 students could correctly determine instantaneous speed on a linear part of a distance-time graph that went through the origin (question 5 on his TUG-K test), but only $21 \%$ could do this on a linear part of a distance-time graph that did not go through the origin (question 17 on the TUG-K test). In the latter case, almost half the students calculated speed as the ratio of distance over time.

Likewise, when asked to determine the speed from a distance-time graph, our students tend to use the formula

$$
v=\frac{d}{t}
$$

which they often interpret as

$$
\text { speed equals distance over time, }
$$

rather than

average speed equals change in distance over change in time;

hence many divide distance by time. Both interpretations can be found, often side by side, in Irish secondary school textbooks (almost in the same breath as the definition of speed as a unit distance). Almost all distance-time graphs our students have interacted with in school are straight lines through the origin [25]; thus they may obtain a value for speed from a distance-time graph without realizing that there is a difference between interpretations (3) and (4), let alone that (3) is an incorrect interpretation of Eq. (2).

If Elby's WYSIWYG notion applies to distance-time graphs, the compelling visual attribute of a distance-time graph that does not go through the origin would cue a productive response (that the speed is constant). However, paired with our students' prior experience, it could also cue an unproductive response (dividing the two coordinates no longer yields a correct value for the speed).

\section{RESEARCH DESIGN}

The research in the literature described above has, in the main, dealt with qualitative reasoning primarily or exclusively. In this paper, we extend these studies by investigating students' qualitative and quantitative reasoning when interpreting numerical distance-time graphs, and whether there are correlations between these types of reasoning.

We were interested in learning more about how students approach the interpretation of these representations and the nature of the difficulties they encounter. We carried out a number of empirical investigations and correlated the results. In addition to the questions on distance-time graphs given at the start of Sec. III, we checked whether students were able to determine the slope at a point in a $y, x$ graph, in an attempt to divorce issues with the physics from technical issues. An example of such a question is given in Fig. 1(b).
We also sought to investigate whether there is a context dependence. As stated in Sec. III, our students had encountered distance-time graphs and problems involving speed, distance, and time earlier in their education. This may impact (positively or negatively) on their approach, and makes it hard to divorce issues with interpreting any straight line slope (and they will have seen straight line graphs through the origin in many different contexts) from issues with the familiar context of distance-time graphs. In an attempt to separate these issues, we asked near-isomorphic questions on closely analogous linear water level versus time graphs. An example is given in Fig. 1(c). We asked whether water is entering or leaving a pool, whether the water level is changing at an increasing, decreasing, or constant rate, and how quickly the water level changes at a particular instant.

We identified three research questions:

(1) What difficulties do students encounter when dealing with a linear distance-time graph, and how prevalent are these difficulties? Specifically,

- What difficulties do students encounter when identifying the direction of motion from a linear distancetime graph, and how prevalent are these difficulties?

- What are the various lines of reasoning students use to assess whether the speed is constant from a linear distance-time graph, and how prevalent are they?

- What difficulties do students encounter when determining a value for the speed from a linear distancetime graph, and how prevalent are they?

- Is the reasoning students use to assess whether the speed is constant from a linear distance-time graph a predictor for how they determine the speed?

(2) To what extent is the ability to determine the slope of a $y, x$ graph and to answer qualitative aspects of numerical distance-time graphs sufficient to successfully determine a value for the speed from a linear distance-time graph?

(3) Do students experience these difficulties to the same extent when they are asked questions on analogous water level versus time graphs?

To answer these research questions, we gave students written pen-and-paper questions concerning a number of linear graphs. They were asked to provide explanations with their answers. Pretests were sometimes administered online, sometimes on paper in the laboratory at the start of week 1 or 2 of a 12-week semester; we found no significant differences in the answers. Post-test results were obtained in written examinations administered in weeks 6 and 12 .

The data were obtained from three to four different cohorts of 50 to 70 students each in any given year, over a period of three years. The students were typically aged 18 , with a small $(<10 \%)$ admixture of mature students. For an estimated $90 \%$ of students, English is the first (native) language; most of the other students also have a good to excellent command of English. All students attended Dublin City University, Ireland, with the intention to study a natural science or life science other than physics. 
About $75 \%$ of them have not studied physics since they were 15 years old; about $20 \%$ have taken physics at high school level. The students were offered three lectures a week in mechanics, including uniform motion, and one 2.5-hour laboratory on a mechanics topic per week. The laboratories were self-contained and more or less independent of the lectures, but the physics topics contained in the labs would typically have been discussed in lecture before the pretest was administered.

Each cohort was given two different pretest or post-test questions. For example, a particular student cohort may have been given a negatively sloped $y, x$ graph and a positively distance-time graph in the same pretest. That same cohort would then have been given a positively sloped $y, x$ graph and a positively water level versus time graph in the same post-test.

Individual answers from written questions, discussions in the laboratory, and semistructured interviews were analyzed. The data were then made quantitative by categorizing the answers [26]. The categories were not predetermined, but emerged from studying the students' answers. Eventually we only needed a small number of categories that proved stable across the different contexts ( $y, x$ graphs, distance-time graphs, water level versus time graphs) for which we asked questions. This categorization allowed us to describe the many answers collectively. We achieved intrarater and interrater reliability of over $90 \%$ consistently.

As the prevalence of different lines of reasoning in different contexts within a large group of students was the main concern of this study, most information on students' reasoning was obtained from their written explanations to their responses. In the majority of cases, their explanations were sufficiently clear to allow categorization with a great degree of certainty. Our interpretations were substantially corroborated in a small number of semistructured interviews and many informal discussions with students within the laboratory. Unless stated otherwise, the quotes cited and our interpretations thereof are derived from the written responses.

Though we present both pretest and post-test data, the focus of this paper is not on the intervention (an inquirybased laboratory). The main reason for including both sets of data is to justify the categories identified, and to give the reader an appreciation for the stability of both the categories and the students' difficulties. In fact, one category would not have emerged as important from analysis of the pretest data alone; only the post-test data justified its existence as a separate category.

\section{IDENTIFICATION OF STUDENT DIFFICULTIES WITH LINEAR DISTANCE-TIME GRAPHS AND THEIR PREVALENCES}

As stated at the start of Sec. III, we investigated the extent to which students make sense of linear distance time graphs by asking them to determine the direction, constancy, and speed of the motion. This naturally leads us to split the research question into the subquestions listed above. In this section, we address each of these subquestions in turn.

\section{A. A note on statistics}

We found no significant differences between cohorts when the same questions were asked at the same stage (pre- or postintervention) at the $p=0.01$ level, and almost none at the $p=0.05$ level, measured by $\chi^{2}$ testing. We have found that rounding our results to the nearest $5 \%$ is a simple and effective way to reflect the near constancy of the results between cohorts. For example, if the wholegroup prevalence is $55 \%$, then the prevalence within almost all of the individual cohorts would be between $50 \%$ and $60 \%$.

To safeguard the readability of the paper, we will typically only discuss prevalences to the nearest $5 \%$. However, data are quoted to the nearest $1 \%$ in the tables. (Taking into account the high but not perfect interrater reliability, we feel that the true accuracy is probably closer to the 5\% than the $1 \%$ level.) When we say a difference is significant, we mean at the $p=0.05$ level as measured by $\chi^{2}$ testing. Only when necessary do we report on the more detailed statistical analysis we carried out.

\section{B. Determining the direction of motion}

The pretest questions on distance-time graphs used a graph like that of Fig. 1(a); some with positive slope $(n=356)$, others with negative slope $(n=194)$. We ascertained that students have some difficulties in determining in which direction the ball moves. Some $20 \%$ of 550 students stated that insufficient information was given, and claimed they needed to know where the reference point was (or complained that it was not plotted on the graph). This is another example of a difficulty that is obscured by asking questions on graphs that go through the origin only: in that case, the reference point is automatically the position at $t=0$, and there is no need to mention a reference point.

Second, we found a difference between positively and negatively sloped graphs. For positively sloped graphs, $20 \%$ of students wrote that the ball was moving towards the reference point, while for negatively sloped graphs only $10 \%$ wrote that the ball moved away from it. The incorrect answers seem to be due to confusion about the reference point. Discussions and interviews with students helped us to ascertain that the students typically had no difficulty with the wording of the question; the difficulty was with representing the reference point in the graph, which may be interpreted as a kind of graph-as-picture error. The most common explanation for choosing the wrong answer was, for both types of graph, something like

The distance is increasing and if it is moving towards a point it will get there when at the right distance. 
In other words, $P$ is assumed to be at the end point of the graph. We also find that many students give explanations like

We are told that it moved to a fixed point $P$

or

As $P$ is a fixed point the ball will move directly towards $P$.

Neither statement is true, and it is interesting to see how students try and incorporate the unfamiliar mention of a reference point. More importantly, this kind of answer could remain hidden in questions on negatively sloped graphs, as students obtain the correct answer with incorrect reasoning. In fact, we feel that this explains most of the different prevalences in assigning a wrong direction to the motion of the ball for positively and negatively sloped graphs. Slope-height confusion does not appear to be prevalent at all.

In summary, we have found that only two-thirds of students could determine the direction of motion from the distance-time graph in the problem presented to them. Most of the difficulties seem to stem from the unfamiliar mention of a reference point; even though students typically had no problem with the fact that there has to be a reference point ("zero") somewhere, they were unsure how the graph showed this. Slope-height confusion did not appear to play a significant role.

\section{Determining whether the speed is constant}

As Table I shows, $75 \%$ of 550 students stated that the speed of the ball was constant. The graph-as-picture error rarely seems to occur in this context: we think students making this error would answer, e.g., that the speed increases in the negatively sloped graph (as the ball would roll down the hill). A detailed study of students' responses confirms this impression.

At first glance, it may appear that the $25 \%$ who stated that the speed changed made a height-for-slope error, for example, by interpreting a decreasing distance as a decreasing speed. However, this appears to be true only for two-fifths of this group ( $10 \%$ of the overall population); and typically, their explanations do not allow us to get an impression of whether this is a coherent slope-for-height error or an almost instantaneous minigeneralization [22]. Three-fifths (15\% of the overall population) calculated the speed at two or more instants using the incorrect interpretation (3) consistently, and based their answers on these calculations.

The $75 \%$ of answers stating that the speed of the ball is constant divides into four categories, one of which we divide into two subcategories. The first category $35 \%$ of 423 students) used reasoning based on intervals as exemplified below. The category can be divided into two subcategories. The first subcategory (68 students) use qualitative or semiqualitative reasoning only, e.g.,

The ball moves the same distance in the same time each time

or

At each time interval the distance has increased by $3 \mathrm{~m}$.

Sometimes, the reasoning does not provide evidence for thinking in terms of covariation, but still involves an interval:

Distance increases by $2 \mathrm{~m}$.

TABLE I. Pretest results for the question of whether the speed of the ball is increasing, decreasing, or constant. Correct answers (constant speed) are categorized by reason (straight line or constant slope or interval reasoning, both or other). The first numerical column gives percentages of the 356 students who took a pretest involving a graph with positive slope; likewise the second numerical column for negatively sloped graphs, and the third column for all students combined. The fourth numerical column shows percentages only for students who took the $y, x$ graph pretest as well as the distance-time graphs and answered it correctly. The fifth column shows percentages only for students who also correctly determined the direction of motion. Note that in many, but not all sets of questions, students were also asked to state whether the speed was increasing or decreasing. This information is given in the bottom part of the table. In all tables, correct answers are printed in bold.

\begin{tabular}{|c|c|c|c|c|c|}
\hline & $\begin{array}{l}\text { Positive slope } \\
\text { Pretest } \\
(n=356)\end{array}$ & $\begin{array}{c}\text { Negative slope } \\
\text { Pretest } \\
(n=194)\end{array}$ & $\begin{array}{c}\text { All } \\
\text { Pretest } \\
(n=550)\end{array}$ & $\begin{array}{l}\text { Context-free } \\
\text { Correct } \\
(n=175)\end{array}$ & $\begin{array}{c}\text { All prep } \\
\text { Correct } \\
(n=123)\end{array}$ \\
\hline $\begin{array}{l}\text { Constant speed (total) } \\
\text { Straight line or constant slope } \\
\text { Interval reasoning } \\
\text { Slope and interval } \\
\text { Other (e.g., proportional reasoning) }\end{array}$ & $\begin{array}{c}79 \%(282) \\
39 \%(138) \\
28 \%(98) \\
2 \%(6) \\
11 \%(40) \\
(n=198)\end{array}$ & $\begin{array}{c}73 \%(141) \\
39 \%(75) \\
21 \%(41) \\
1 \%(2) \\
12 \%(23) \\
(n=194)\end{array}$ & $\begin{array}{c}77 \%(423) \\
39 \%(213) \\
27 \%(147) \\
1 \%(8) \\
11 \%(63) \\
(n=392)\end{array}$ & $\begin{array}{l}\mathbf{7 8 \%}(\mathbf{1 3 7}) \\
39 \%(68) \\
30 \%(52) \\
1 \%(1) \\
9 \%(16)\end{array}$ & $\begin{aligned} & 83 \%(102) \\
& 43 \%(53) \\
& 29 \%(36) \\
& 1 \%(1) \\
& 10 \%(12)\end{aligned}$ \\
\hline $\begin{array}{l}\text { Speed increases } \\
\text { Speed decreases } \\
\text { Do not know or no answer }\end{array}$ & $\begin{array}{l}10 \%(16) \\
10 \%(23) \\
\approx 0 \%(5)\end{array}$ & $\begin{array}{l}\approx 0 \%(3) \\
25 \%(49) \\
\approx 0 \%(1)\end{array}$ & $\approx 0 \%(6)$ & & \\
\hline
\end{tabular}


The other subcategory, consisting of 79 students, explicitly and correctly at least indicated all numerical values required to calculate the slope (and hence the speed), e.g.,

Traveling the same amount of distance at the same amount of time, i.e., $3 \mathrm{~m}$ every $5 \mathrm{~s}$

or gave the result of the calculation:

The ball moves at $0.6 \mathrm{~m} / \mathrm{s}$ the whole time.

The subdivision seemed useful, as we expected students who gave correct numerical values to be less likely to obtain an incorrect value for the speed at a given instant.

The second and largest category $(50 \%)$ stated that the slope is constant, or that the line is straight, e.g.,

The slope of the line does not change

or

The graph is straight, therefore speed is constant.

The third category comprises students who responded in terms of both intervals and slope. For pretest questions, this category is almost unpopulated, but in post-tests they make up some $10 \%$ of the answers; for the sake of consistency, we have included this category in the pretest analysis also.

The fourth category contains all other answers. These include students who stated they used proportional reasoning and those who gave an unclear or no explanation.

In summary, three-quarters of our students are able to determine correctly that a linear distance-time graph represents motion at constant speed, be it by interval reasoning, considering the shape of the graph, or by some other means. Taking the ratio of the coordinates at two different points on the graph, and observing that these values are different, is the most common reason for obtaining an incorrect answer. This indicates that incorrect prior learning is likely to have played a role.

\section{Determining the value of the speed}

Just under $20 \%$ of all students were able to correctly determine a correct value for the speed from the distancetime graph; just over $20 \%$ of students who determined that the speed of the ball is constant could. These figures were obtained after purely calculational errors, read-off errors, and sign errors are excused. The most prevalent answer, given by just over half of the students, was obtained by calculating the speed using interpretation (3). All of these numbers closely match the findings of Beichner's study of U.S. students [24]. Unlike the context-free case, none of these students appear to have used the origin erroneously as one of the two points in an otherwise correct calculation.

Just over $15 \%$ of the students gave the value for the distance they read off the graph (sometimes in units of $\mathrm{m}$, sometimes in $\mathrm{m} / \mathrm{s}$ ). This appears to be an example of some students getting confused between distance and speed in an educational context. Typically, such answers are not explained, other than by a description of how the value was read off the graph. However, when we probed further, these students did not reveal an inability to distinguish between height and slope. Rather, they did not know how to obtain a value for the speed, and simply gave the best (or only) answer they could think of. New issues regarding speed came to light. Some volunteered that they did not understand the question, as they did not think there is such a thing as speed at an instant; they appeared to struggle with Zeno's arrow paradox. It is interesting to note that these students did not give zero speed as an answer, as might be expected. We will not delve deeper into this interesting find in this paper, but intend to investigate this further in the future.

\section{E. Reasons for constancy of speed as a predictor}

We have correlated the answers to the questions on constancy and numerical value of the speed and present a summary in Table II. Prior to further analysis of the students' answers, we would have deemed interval reasoning a more satisfactory explanation, because this explicitly reveals a

TABLE II. Pretest results linking the reasons students gave for correctly identifying constant speed with how they determined a value for the rate of change at one particular instant. For example, the fourth column shows that of the 147 students (i.e., $35 \%$ of all students who identified that the speed was constant) who used interval reasoning to determine that the speed changes at constant rate in the pretest, $21 \%$ correctly determined the rate of change using a correct method, $52 \%$ divided the two coordinates, $20 \%$ read off the value, and $10 \%$ gave uncategorized answers or no answers.

Interval reasoning only

Approaches to correctly

identifying constant speed

Qualitative Quantitative

All

Slope only

Slope + interval

Other

Total

Approaches to finding

$n=68$

$n=79$

$n=147$

$n=213$

$n=8$

$n=55$

$n=423$

instantaneous speed

(16\%)

(19\%)

(35\%)

$(50 \%)$

(2\%)

(15\%)

(100\%)

$\Delta y / \Delta x$ or similar

9\% (6)

$32 \%(25)$

21\% (31)

$21 \%(45)$

$38 \%(3)$

$15 \%(8)$

$21 \%(87)$

Read off

$24 \%(16)$

$13 \%(10)$

$52 \%(76)$

$58 \%$ (124)

$50 \%$ (4)

$36 \%(20)$

$0 \%(0)$

$31 \%(17)$

$53 \%(224)$

Other or no answer

$15 \%(10)$

$5 \%(4)$

$10 \%(14)$

$7 \%(15)$

$13 \%(1)$

$18 \%(10)$

$17 \%(72)$

$9 \%(40)$ 
TABLE III. Pretest results for the task to determine the slope at a point on a straight line $y, x$ graph not through the origin. The row labeled $y / x$ includes answers in which students stated they calculated $\Delta y / \Delta x$, but used $(0,0)$ as one of the points or $(1,0)$ and $(0,3)$ as the two points. The row labeled "interval reasoning" excludes students who used $\Delta y / \Delta x$.

\begin{tabular}{|c|c|c|c|}
\hline $\begin{array}{l}\text { Method of } \\
\text { calculating slope }\end{array}$ & $\begin{array}{c}\text { Positive slope } \\
\text { Pretest } \\
(n=206)\end{array}$ & $\begin{array}{c}\text { Negative slope } \\
\text { Pretest } \\
(n=205)\end{array}$ & $\begin{array}{c}\text { All } \\
\text { Pretest } \\
(n=411)\end{array}$ \\
\hline$\Delta y / \Delta x$ & $52 \%(107)$ & $46 \%(94)$ & $49 \%(201)$ \\
\hline Formula and 2 points & $19 \%(39)$ & $20 \%(41)$ & $19 \%(80)$ \\
\hline Formula & $14 \%(29)$ & $15 \%(30)$ & $14 \%(59)$ \\
\hline Interval reasoning & $4 \%(9)$ & $4 \%(9)$ & $4 \%(18)$ \\
\hline$y / x$ & $18 \%(37)$ & $24 \%(50)$ & $21 \%(87)$ \\
\hline Read off & $3 \%(7)$ & $4 \%(8)$ & $4 \%(15)$ \\
\hline Other & $15 \%(30)$ & $11 \%(22)$ & $13 \%(52)$ \\
\hline No answer & $12 \%(25)$ & $15 \%(31)$ & $14 \%(56)$ \\
\hline
\end{tabular}

correct reasoning process, while students in the other categories may have relied on identifying a surface feature or may have been uncertain of their answer. To our surprise, we found that the method by which students find a numerical value for the speed at an instant is independent of the reasoning by which they determined that the speed was constant. (By contrast, less than 2\% of students who said the speed was not constant determined the speed correctly.)

Incredibly, $50 \%$ of the students who gave a numerical interval-based explanation for the constancy of the speed calculated the speed at an instant using (3). These are not just the students who gave the correct magnitude of individual distance and time intervals: some students write in the first question that the speed is $0.6 \mathrm{~m} / \mathrm{s}$ throughout, and in the second that it is $0.8 \mathrm{~m} / \mathrm{s}$ at a particular instant. When probed, many of them justify the difference by labeling the former "average speed" and the latter "instantaneous speed", but (thankfully) often lack conviction in doing so. While one should always be careful not to overinterpret an incorrect answer, the conclusion seems inescapable that for these students at least there is nothing wrong with their distance-time graph interpretation; but they allow prior learning of the wrong definition of (average) speed to override their correct graphical interpretation.

\section{IS QUALITATIVE UNDERSTANDING AND TECHNICAL ABILITY ENOUGH?}

To gain deeper insight into the findings of the previous section, we investigated whether students had difficulty determining the slope of any graph, to decouple any issues with the physics from mathematical or technical issues. We also investigated whether students who were able to successfully determine the slope of a $y, x$ graph at a point and able to determine the direction and constancy of the speed from the graph did significantly better in determining a value for the speed.

\section{A. Context-free graphs}

We asked students to calculate the slope of a line at a particular point in graphs like those of Fig. 1(b). The results of our analysis are given in Table III. We find no significant difference between graphs with positive $(n=206)$ and negative $(n=205)$ slopes.

Just under $50 \%$ of 411 students correctly calculated the slope. About $40 \%$ of those answers (hence $20 \%$ of all students) mentioned both a formula and taking two points:

The slope is the same at all points. To measure the slope we take 2 points on the line and apply the formula $m=(y 2-y 1) /(x 2-x 1)$. Using two points $(2,3)$ and $(3,6)$ we determine $m=3$

using the point $(1,3)$ and $(0,5)$ I was able to work out the slope.

Using slope formula taking 2 points on the line I could work out the slope and it is negative as it is going down

A further $30 \%$ of the correct answers just stated the formula or that they used one:

Used the slope formula to determine the slope,

$$
\operatorname{Rise}(y 2-y 1) / \operatorname{run}(x 2-x 1) \text {. }
$$

Only $10 \%$ of those answers, or $5 \%$ of the total, used interval reasoning:

The slope of a line is constant and in this case it is simply -2 . With every 1 unit increment on the $x$ axis there is

2 units decline on the $y$ axis.

The first quote explicitly states the idea that the slope is the same at all points. Only 9 students out of 411 did so.

The incorrect answers can be split into three categories. Just over $20 \%$ calculate $y / x$. One-quarter of these students used the correct equation (1), but either took $(1,3)$ and $(0,0)$ 
or $(1,0)$ and $(0,3)$ as the two points. Another quarter of these students explicitly stated that slope equals $y / x$.

Just under $5 \%$ of students gave both coordinates of the point as their answer, typically written as " $(1,3)$ " or " 1,3 "; two students wrote down the $y$ coordinate only. Just under $15 \%$ did not give a value. In the written answers, $10 \%$ gave no explanation or said that they do not know how to do this. Informal discussions in the laboratory suggest that about half of these students had forgotten how to calculate the slope of a line, while the other half could, but did not know how to find the value for the slope at a point. This does not appear to be a Zeno's arrow paradox problem, but simply a matter of never having been asked to think of the slope at a point on a straight line.

Thus, a maximum of $10 \%$ of answers could be said to indicate possible slope-height confusion if we are willing to consider all of the students who did not know that a straight line has a slope at a point and all of the students who gave one or both coordinates as showing slope-height confusion. From this we may conclude that this is not a common difficulty for our student cohorts.

The results on $y, x$ graphs strongly suggest that many students had difficulty in determining the slope of a line. Most responses, whether correct or incorrect, appeared to rely on the use of a formula; only some 5\% of answers comprised interval reasoning. A negligible number of students explicitly stated that the slope of a straight line is equal to the slope at a point.

\section{B. Context-free graphs as a predictor}

A total of 351 students participated in both of the pretest questions on $y, x$ graphs and distance-time graphs. This allows us to have a detailed look at any correlations between the answers. Of these 351 students, 175 students answered the $y, x$-graph question correctly. Within this subgroup, we also looked at the 102 students who also answered the questions on the direction and constancy of the motion correctly.

The fourth and fifth columns of Table I show that there was no difference between these subgroups of students and the entire group when it comes to determining whether the speed of the ball is constant or not; neither in the number of correct determinations nor in the reasoning used. It is therefore reasonable to conclude that the reasoning used by students to determine whether the speed of an object is constant from a distance-time graph is independent of their ability to determine the value of a slope correctly.

One-quarter of the 175 students who correctly determined the slope at a point in a $y, x$ graph successfully determined a value for speed. As shown in Table IV, even within the subgroup of 102 students who also answered the questions on the direction and constancy of the motion correctly, only $35 \%$ gave a correct numerical value.

These findings strongly suggest that merely focusing on preparing the students optimally, in terms of them being able to calculate the slope of a graph and being able to interpret the qualitative aspects of a numerical distancetime graph, will not suffice to achieve more than one-third of our students to correctly determine the speed from a linear distance-time graph.

\section{WATER LEVEL VERSUS TIME GRAPHS}

Having concluded that possible technical issues alone cannot explain the low fraction of students who can correctly determine a value for the speed from a linear distance-time graph, we also investigated a closely analogous question on water level versus time graphs. If Elby's WYSIWYG idea is dominant, then students should answer the water level questions more or less the same as the distance-time questions; if not, then context dependence is more important.

The categorization found for distance-time graphs proved useful in this case again. A typical example of a student using interval reasoning was

The water level changes at a constant rate as the intervals are the same.

Answers based on the shape of the graph use reasoning like

The water level is changing at a constant rate as it is a straight line graph which shows this.

TABLE IV. Pretest results linking the reasons students gave for correctly identifying constant speed with how they determined a value for the rate of change at one particular instant. Shown here are results only for the students who answer the questions on $y$, $x$ graph, direction and constancy of motion correctly. For an example of how to read the table, see the caption of Table II.

\begin{tabular}{|c|c|c|c|c|c|c|c|}
\hline \multirow[b]{2}{*}{$\begin{array}{l}\text { Approaches to correctly } \\
\text { identifying constant speed }\end{array}$} & \multicolumn{3}{|c|}{ Interval reasoning only } & \multirow[b]{2}{*}{ Slope only } & \multirow[b]{2}{*}{ Slope + interval } & \multirow[b]{2}{*}{ Other } & \multirow[b]{2}{*}{ Total } \\
\hline & Qualitative & Quantitative & All & & & & \\
\hline $\begin{array}{l}\text { Approaches to finding } \\
\text { instantaneous speed }\end{array}$ & $\begin{array}{l}n=16 \\
(16 \%)\end{array}$ & $\begin{array}{l}n=20 \\
(20 \%)\end{array}$ & $\begin{array}{c}n=36 \\
(35 \%)\end{array}$ & $\begin{array}{l}n=53 \\
(52 \%)\end{array}$ & $\begin{array}{c}n=1 \\
(1 \%)\end{array}$ & $\begin{array}{l}n=12 \\
(12 \%)\end{array}$ & $\begin{array}{c}n=102 \\
(100 \%)\end{array}$ \\
\hline$\Delta y / \Delta x$ or similar & $13 \%(2)$ & $50 \%(10)$ & $33 \%(12)$ & $38 \%(20)$ & $0 \%(0)$ & $33 \%(4)$ & $35 \%(36)$ \\
\hline$y / x$ or similar & $57 \%(9)$ & $35 \%(7)$ & $44 \%(16)$ & $45 \%(24)$ & $100 \%$ & $50 \%(6)$ & $46 \%$ (47) \\
\hline Read off & $19 \%(3)$ & $5 \%(1)$ & $11 \%(4)$ & $6 \%(3)$ & $0 \%(0)$ & $17 \%(2)$ & $9 \%(9)$ \\
\hline Other or no answer & $13 \%(2)$ & $10 \%(2)$ & $11 \%(4)$ & $11 \%(6)$ & $0 \%(0)$ & $0 \%(0)$ & $10 \%(10)$ \\
\hline
\end{tabular}


Finally, we give a typical example of an answer showing both slope and interval reasoning, and yet an incorrect determination of the rate of change:

The water level is changing at a constant rate. For every $100 \mathrm{~s}$ the water level decreases by $40 \mathrm{~m}$. This situation leads to a straight line graph when it is graphed. Both the straight line graph and the same decrease in water level per $100 \mathrm{~s}$ indicate a constant rate of change of water level of pool.

$$
\begin{aligned}
& S=\text { speed. } D=\text { distance } / \text { displacement. } \\
& T=\text { time. } \\
& S=D / T=\frac{160}{200}=\text { speed of } 0.8 \mathrm{~cm} / \mathrm{s} .
\end{aligned}
$$

The pretest questions on water level versus time graphs used a graph like that of Fig. 1(c). The first question took the form of asking students whether water was flowing into the pool, out of the pool, or not flowing at all. Over $95 \%$ of answers were correct, for both positively and negatively sloped graphs. Although the context or the phrasing of the question here is evidently sufficiently different from the distance-time graphs to trigger a different response, the answers were consistent with our earlier interpretation that the incorrect answers to the corresponding question in distance-time graphs were not due to slope-height confusion. The data were also consistent with our finding that difficulties with interpreting the reference point were an obstacle for students when interpreting distance-time graphs; in this case, the bottom of the pool is the (implicit) known reference point.

As with distance-time graphs, we found no significant difference between graphs with positive or negative slopes; but in this case, over $95 \%$ of students stated that the filling rate was constant, as shown in Table V. These numbers were significantly different from those obtained for the distance-time graphs; in fact, many more students answered correctly in this context, which they had not encountered before in a formal setting. This is a further indication that context is an important factor, given the close relation between the water level versus time graph and the distance-time graph. Indeed, many students spontaneously used the word speed when describing the pool filling or emptying; and yet, this question did not appear to trigger students taking two points from the graph to work out the speed at two points.

The close similarity between the water level versus time and distance-time graphs allows us to verify Elby's suggestion [22] that the dominant feature of the graph is very influential on students' answers. The different prevalences in the two contexts provided an empirical indication that the dominant feature of the graph may have triggered initial student responses, but it was not what determined the final response. Furthermore, we have asked the questions with and without a line through the data points; no differences in prevalences were observed, even though the "visual dominance" of the constancy of the slope would change between the two situations. We therefore conclude that a constancy cue provided by a straight line does not explain our students' answers.

A correlation between the reasoning used to conclude the filling rate is constant and the reasoning used to find how quickly the pool fills at a given instant is given in Table VI. Two important findings emerge:

- Just over $40 \%$ of students correctly calculated the rate of change of the water level, compared to $20 \%$ who correctly calculated the speed from distancetime graphs. The numerical answers were independent of the method used to determine that the filling rate is constant.

- A far greater fraction of students now applied interval reasoning (60\% versus $35 \%$ ), even though this pretest question was asked at the same stage in the course as the distance-time graph pretest question. As we deem an explanation based on the straightness of the line more likely to be a pat answer than an explanation based on interval reasoning, we infer from this that our students engaged with the water level versus time graph more than with the distance-time graph.

It is worth commenting on the different categorization of incorrect answers, which we ascribe to the way the

TABLE V. Pretest results for the question whether the water level is changing at an increasing, decreasing, or constant rate. No significant difference was found between positively and negatively sloped lines.

\begin{tabular}{lccc}
\hline \hline & Positive slope & Negative slope & All \\
& Pretest $(n=179)$ & Pretest $(n=164)$ & Pretest $(n=343)$ \\
\hline Constant rate (total) & $\mathbf{9 6 \%}(\mathbf{1 7 2})$ & $\mathbf{9 9 \%}(\mathbf{1 6 2})$ & $\mathbf{9 7 \%}(\mathbf{3 3 4})$ \\
Straight line or constant slope & $\mathbf{3 6 \% ( 6 5 )}$ & $\mathbf{4 5 \%}(\mathbf{7 3})$ & $\mathbf{4 0 \%}(\mathbf{1 3 8})$ \\
Interval reasoning & $\mathbf{4 9 \%}(\mathbf{8 7})$ & $\mathbf{4 5 \% ( 7 3 )}$ & $\mathbf{4 7 \%}(\mathbf{1 6 0})$ \\
Slope and interval & $\mathbf{3 \%}(\mathbf{6})$ & $\mathbf{1 \%}(\mathbf{1})$ & $\mathbf{2 \%}(\mathbf{7})$ \\
Other (e.g., proportional reasoning) & $\mathbf{8 \%}(\mathbf{1 4})$ & $\mathbf{7 \%}(\mathbf{1 1})$ & $\mathbf{7 \%}(\mathbf{2 5})$ \\
Rate increases & $0 \%(0)$ & $0 \%(0)$ & \\
Rate decreases & $0 \%(0)$ & $0 \%(0)$ & $4 \%(13)$ \\
Do not know or no answer & $4 \%(7)$ & $4 \%(6)$ & \\
\hline \hline
\end{tabular}


TABLE VI. Pretest results linking the reasons students gave for correctly identifying constant rate with how they determined a value for the rate of change at one particular instant. For an example of how to read the table, see the caption of Table II.

\begin{tabular}{|c|c|c|c|c|c|c|c|}
\hline \multicolumn{8}{|c|}{ Interval reasoning only } \\
\hline $\begin{array}{l}\text { Approaches to correctly identifying } \\
\text { constant rate of change }\end{array}$ & Qualitative & Quantitative & All & Slope only & Slope + interval & Other & Total \\
\hline $\begin{array}{l}\text { Approaches to finding } \\
\text { instantaneous rate of change }\end{array}$ & $\begin{array}{c}n=91 \\
(33 \%)\end{array}$ & $\begin{array}{l}n=62 \\
(22 \%)\end{array}$ & $\begin{array}{l}n=153 \\
(55 \%)\end{array}$ & $\begin{array}{c}n=89 \\
(32 \%)\end{array}$ & $\begin{array}{l}n=7 \\
(3 \%)\end{array}$ & $\begin{array}{l}n=30 \\
(11 \%)\end{array}$ & $\begin{array}{l}n=279 \\
(100 \%)\end{array}$ \\
\hline$\Delta y / \Delta x$ or similar & $44 \%(40)$ & $37 \%(23)$ & $41 \%(63)$ & $46 \%(41)$ & $43 \%(3)$ & $37 \%(11)$ & $42 \%(118)$ \\
\hline$y / x$ or similar & $15 \%(14)$ & $18 \%(11)$ & $16 \%(25)$ & $12 \%(11)$ & $0 \%(0)$ & $7 \%(2)$ & $14 \%(38)$ \\
\hline Other & $32 \%(29)$ & $18 \%(11)$ & $26 \%(40)$ & $22 \%(20)$ & $0 \%(0)$ & $33 \%(10)$ & $25 \%(70)$ \\
\hline No answer & $9 \%(8)$ & $27 \%(17)$ & $16 \%(25)$ & $19 \%(17)$ & $57 \%(4)$ & $23 \%(7)$ & $19 \%(53)$ \\
\hline
\end{tabular}

question was asked. Unlike distance-time graph questions, we asked students first what the water level was at a particular instant, before asking about the rate of change at the same instant. In the light of the discussion above, one would now expect that students who read off the value from distance-time graphs would be confused; this is how we explain the absence of the "read-off" category and the significant increase in the number of no answers.

\section{SUMMARY OF PRETEST RESULTS}

The pretest results allow us to give a provisional answer to the research questions asked.

(1) Difficulties with linear distance-time graphs may be summarized as follows:

- About $20 \%$ of our students claim they need to know the position of the reference point to determine the direction of motion; they do not seem to realize that the reference point is at $d=0$ by definition. This may be interpreted as a graph-as-picture error, in that these students expect the graph to give an iconic representation of the reference point. Another $10 \%$ to $20 \%$ took the reference point to be the end point of the graph. Sixty to $70 \%$ of students correctly identified the direction of the motion. Slope-height confusion did not seem to play a significant role.

- $75 \%$ of our students correctly assess that the speed is constant in a linear distance-time graph; $25 \%$ use interval reasoning, $40 \%$ slope reasoning, $10 \%$ other reasoning. The $25 \%$ of incorrect answers are split into two categories: those who divide two coordinates and different points (15\%) and those who misidentify an increasing (decreasing) distance with an increasing (decreasing) speed (10\%).

- Only $20 \%$ of our students correctly determine a value for the speed from a linear distance-time graph. 50\% divide the coordinates, and $15 \%$ quote the distance value.

- The approach our students take to determine the value for the speed from a linear distance-time graph is independent from the reasoning they use to assess whether the speed is constant.
(2) The ability to determine the slope of a $y, x$ graph and to answer qualitative aspects of numerical distancetime graphs is not sufficient to successfully determine a value for the speed from a linear distance-time graph, as only $35 \%$ of students who both correctly determine the slope at a point on a linear $y, x$ graph, the direction of motion and the constancy of speed on a linear distance-time graph correctly determine the speed. We conclude that merely making sure students can do all the reasoning and acquire all relevant techniques is insufficient to get a satisfactory fraction of students to determine the speed correctly.

(3) When comparing the answers from the water level versus time graphs with those for distance-time graphs, it emerges that students are more likely to answer the questions correctly for water level versus time graphs, and that they are more likely to use interval reasoning in the water level versus time setting, both when determining whether the rate of change is constant and in determining a numerical value for the slope.

Even though the questions are not exactly isomorphic, we feel that they are sufficiently similar that the results can be compared. It is striking that students consistently do better in the setting that they have not encountered in an educational context before. To us these findings strongly suggest that prior learning has impacted negatively on how students approach distance-time graphs. As students have no memory of water level versus time graphs, they must engage with the problem to some extent to obtain an answer, while distance-time graphs may trigger a rote procedure (typically, one that yields the correct answer for graphs through the origin only).

We have interviewed 15 students with a view to gaining more insight into these differences, but with little result. Students were generally able to explain the procedure they followed, but struggled to explain why they followed the procedure they chose. One tentative conclusion is that students did not think very much about choosing a procedure; it seemed to be more a reflex than a well-considered choice [27]. Students did not spontaneously see an inconsistency if they chose a correct procedure for $y, x$ graphs but not for distance-time graphs or for water level versus 
time graphs; it appears that the two questions cued different responses.

\section{INSIGHTS FROM POST-TEST ANALYSIS}

We extended the study by asking the same cohorts of students similar but different questions on a post-test following an intervention that focused on interval reasoning. For example, a particular student cohort might have been given a negatively sloped $y, x$ graph and a positively sloped water level versus time graph in the pretest, followed by a positively sloped $y, x$ graph and a negative sloped distancetime graph in the post-test.

As stated before, the aim of this section is not to show the merit of the intervention, but to verify the validity of the pretest analysis. As it turns out, the post-test results were sufficiently different that they provided a rich source of complementary data.

\section{A. Intervention: A uniform motion laboratory}

We have developed three laboratories, each of 2.5 hours, where students are led to focus on interval reasoning. All three use a guided inquiry method. The last two concern the motion of a pendulum and nonuniform motion of a ball, and they are not discussed in detail here. We mention them because they are likely to contribute to students getting used to graph interpretation through interval reasoning.

In the first of these labs, the uniform motion laboratory, we ask the students to carry out three investigations and two tutorial-type questions that allow them to become familiar with and understand a simple approach to breaking down the motion of an object into relevant intervals.

We considered adopting a high-tech design (using, e.g., motion sensors and graphing calculators), but eventually opted to design and implement a low-tech approach: tracks, marbles, and stopwatches. Arguments for and against use of technology are well known [6,8,28-32]. Part of the reason for our choice was financial: we need to cater for 36 pairs of students at a time, so the cost factor is significant. A second factor was the uniform motion laboratory is part of a set of 18 laboratories that are all based around the philosophy that introductory level laboratories should not have black boxes. A final argument was the fact that we feel that students should construct some graphs by hand while being asked about them.

The first experimental task uses the same setup as the opening experiment of the kinematics curriculum in Physics by Inquiry [33]. Students obtain uniform motion

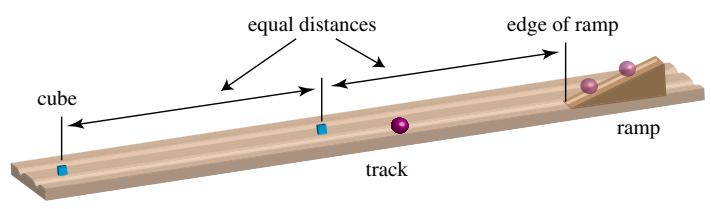

FIG. 2 (color online). Initial setup used in the uniform motion laboratory.

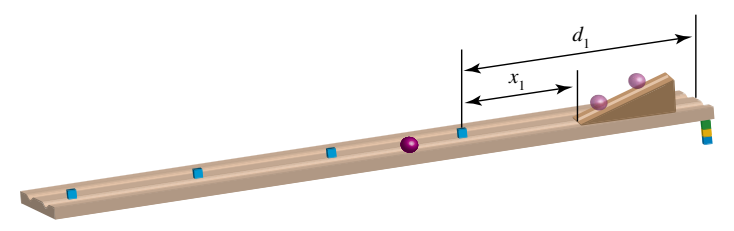

FIG. 3 (color online). The track is divided into four equal lengths. The motion of the ball is considered from the instant the ball reaches the bottom of the ramp. Distances are measured from each cube $i$ both to the end of the track $\left(d_{i}\right)$ and to the bottom of the ramp $\left(x_{i}\right)$.

by measuring the time it takes to traverse the first and second halves of a track whose tilt they adjust, as shown in Fig. 2. Students then use essentially the same setup but now divide the track into four equal parts, as shown in Fig. 3.

The students are instructed to use two separate variables to record the position of the ball: $x_{i}$ for the distance of cube $i$ from the bottom of the ramp, and $d_{i}$ for the distance of cube $i$ from the end of the track. In the experiment, students record the time it takes for the ball to reach each cube, as measured from the time the ball reaches the bottom of the ramp. The students tabulate their data and draw two distance-time graphs, one for $x$ versus $t$ and one for $d$ versus $t$. They are then asked to pick two points, $A$ and $B$, on each graph, and are given a hypothetical student conversation based on real conversations. The conversation gives two written descriptions of approaches to measure speed: by dividing distance by time, or change in distance by change in time. The students are then explicitly asked to write out each calculation using some or all of the variables $x_{A}, x_{B}, t_{A}$, and $t_{B}$, and then using some or all of the variables $d_{A}, d_{B}, t_{A}$, and $t_{B}$. Through Socratic dialogue, the students see that calculating distance over time gives different values for the speed at different times, whereas change in distance over change in time gives a constant
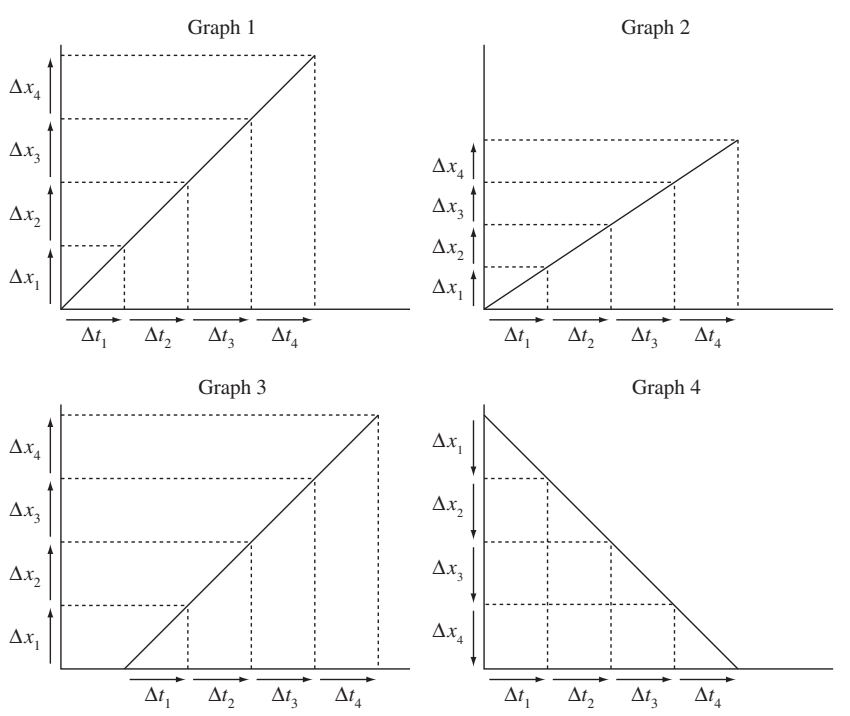

FIG. 4. Four graphs representing uniform motion. 


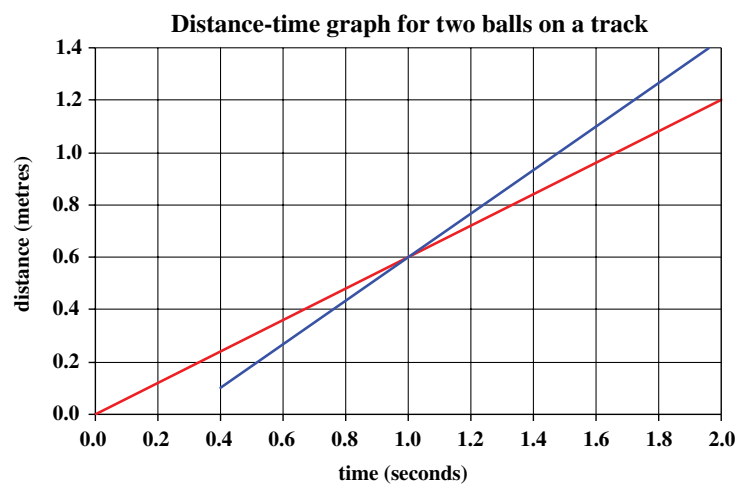

FIG. 5 (color online). Distance-time graph for two balls rolling along two parallel tracks.

value. Although many students strongly hold on to the belief that "speed is distance over time," because "that is the correct formula," most now articulate that dividing the change in distance by the change in time must be a correct method.

We have found it useful to insert two tutorial-type exercises at this point. First, students have to discuss which of the graphs of Fig. 4 represent uniform motion, and they are to determine the speed of a negatively sloped distancetime graph, aided by a hypothetical student conversation. In the third and final investigation, students further strengthen their graph reading skills by recreating the motion of two balls given in the graph of Fig. 5. They are guided to finding the right setup by being asked whether the balls travel at constant speed, at the same speeds, start at the same time; which one finishes first, and whether the balls overtake each other.

\section{B. Distance-time graphs}

Although the prevalences of students' difficulties changed, the same categorization applies. Problems with determining the direction of the motion have virtually disappeared, as over $95 \%$ of students now correctly determined the direction of motion for positively and negatively sloped graphs. Comparing the pretest results from Tables I and II and the post-test results from Tables VII and VIII, we see that while the fraction of respondents stating that the speed is constant remained at $75 \%$, there had been a significant shift in the reasoning used. In the pretest, 35\% of students who said the speed was constant used interval reasoning, but in the post-test $70 \%$ did.

Half of the pretest answers referred only to the shape of the line when determining whether the speed was constant, which drops to $30 \%$ in the post-test. It was encouraging that more students after instruction used interval reasoning not only to analyze the type of motion but also to quantify the speed of motion.

Despite this improvement in qualitative reasoning, only $35 \%$ of all students calculated a correct value of the

TABLE VII. Post-test results for the question whether the speed of the ball is increasing, decreasing, or constant.

\begin{tabular}{|c|c|c|c|}
\hline & $\begin{array}{c}\text { Positive slope } \\
\text { Post-test }(n=112)\end{array}$ & $\begin{array}{c}\text { Negative slope } \\
\text { Post-test }(n=59)\end{array}$ & $\begin{array}{c}\text { All } \\
\text { Post-test }(n=171) \\
\end{array}$ \\
\hline Constant speed (total) & $72 \%(81)$ & $91 \%(53)$ & $82 \%(134)$ \\
\hline Straight line or constant slope & $22 \%(25)$ & $28 \%(16)$ & $25 \%(41)$ \\
\hline Interval reasoning & $39 \%(44)$ & $\mathbf{5 0 \%}(\mathbf{2 9})$ & $45 \%(73)$ \\
\hline Slope and interval & $9 \%(10)$ & $7 \%(4)$ & $9 \%(14)$ \\
\hline Other (e.g., proportional reasoning) & $2 \%(2)$ & $5 \%(3)$ & $3 \%(5)$ \\
\hline Speed increases & $25 \%(29)$ & $\approx 0 \%(1)$ & \\
\hline Speed decreases & $\approx 0 \%(2)$ & $10 \%(5)$ & \\
\hline Do not know or no answer & $0 \%(0)$ & $0 \%(0)$ & $0 \%(0)$ \\
\hline
\end{tabular}

TABLE VIII. Post-test results linking the reasons students gave for correctly identifying constant speed with how they determined a value for the rate of change at one particular instant. For an example of how to read the table, see the caption of Table II.

\begin{tabular}{|c|c|c|c|c|c|c|c|}
\hline \multirow[b]{2}{*}{$\begin{array}{l}\text { Approaches to correctly } \\
\text { identifying constant speed }\end{array}$} & \multicolumn{3}{|c|}{ Interval reasoning only } & \multirow[b]{2}{*}{ Slope only } & \multirow[b]{2}{*}{ Slope + interval } & \multirow[b]{2}{*}{ Other } & \multirow[b]{2}{*}{ Total } \\
\hline & Qualitative & Quantitative & All & & & & \\
\hline $\begin{array}{l}\text { Approaches to finding } \\
\text { instantaneous speed }\end{array}$ & $\begin{array}{l}n=20 \\
(15 \%)\end{array}$ & $\begin{array}{l}n=51 \\
(38 \%)\end{array}$ & $\begin{array}{l}n=71 \\
(53 \%)\end{array}$ & $\begin{array}{l}n=39 \\
(29 \%)\end{array}$ & $\begin{array}{l}n=14 \\
(10 \%)\end{array}$ & $\begin{array}{l}n=10 \\
(7 \%)\end{array}$ & $\begin{array}{c}n=134 \\
(100 \%)\end{array}$ \\
\hline$\Delta y / \Delta x$ or similar & $15 \%(3)$ & $55 \%(28)$ & $44 \%(31)$ & $38 \%(15)$ & $43 \%(6)$ & $40 \%(4)$ & $42 \%(56)$ \\
\hline$y / x$ or similar & $80 \%(16)$ & $41 \%(21)$ & $52 \%(37)$ & $56 \%(22)$ & $57 \%(8)$ & $40 \%(4)$ & $53 \%(71)$ \\
\hline Read off & $0 \%(0)$ & $0 \%(0)$ & $0 \%(0)$ & $0 \%(0)$ & $0 \%(0)$ & $0 \%(0)$ & $0 \%(0)$ \\
\hline Other or no answer & $5 \%$ & $4 \%(2)$ & $5 \%(3)$ & $5 \%(2)$ & $0 \%(0)$ & $20 \%(2)$ & $5 \%(7)$ \\
\hline
\end{tabular}


slope, compared to $15 \%$ in the pretest. If we apply the formula Hake showed to be valid for the Force Concept Inventory [34],

$$
h=\frac{\text { post-test score }- \text { pretest score }}{\text { maximum score }- \text { pretest score }}
$$

we obtain a modest gain of $h=0.21$. And even this modest improvement may paint too optimistic a picture: in an interview conducted after completion of the lab and post-test, one student stated she used the correct formula

Because using two points is more accurate than using one.

As before, students who reasoned based on the shape of the graph were as likely to get the correct numerical value of the slope as those who use interval reasoning.

\section{Technical aspects}

Post-test results for questions similar to that of Fig. 1(b) show two important pieces of information. First, there was a significant difference between positively and negatively sloped graphs. Second, in both cases there was a higher fraction of correct answers, up to over $85 \%$ and $70 \%$, respectively, with high gains of $h=0.69$ and $h=0.49$, respectively. Fewer than $10 \%$ of students now calculated the slope as $y / x$. These results suggest that our teaching of interval reasoning has been successful.

Together with the post-test results for distance-time graphs, these results strongly support the conclusions concerning research question 2 given in Sec. VIII. Even when almost all of our students were able to correctly determine the slope of a $y, x$ graph and infer the direction of motion from a distance-time graph, this resulted in only a modest improvement in determining a value for the speed.

\section{Water level versus time graphs}

The post-test question on water level versus time graphs used graphs like that of Fig. 1(c). A comparison of Tables V and VI with Tables IX and X shows that 95\% of students still stated that the filling rate was constant. Of these students, the fraction whose reasoning was based on the shape of the graph was down from $40 \%$ to $30 \%$, and reasoning based on intervals was up from a total of $50 \%$ to a total of $70 \%$, with a gain of $h=0.45$. The fraction of students who obtained a correct value for the rate at which the water level changes remained static at $40 \%$. The results mirrored those of the distance-time graph post-test questions quite closely.

TABLE IX. Post-test results for the question whether the water level is changing at an increasing, decreasing, or constant rate. No significant difference was found between positively and negatively sloped lines.

\begin{tabular}{|c|c|c|c|}
\hline & $\begin{array}{c}\text { Positive slope } \\
\text { Post-test }(n=112)\end{array}$ & $\begin{array}{c}\text { Negative slope } \\
\text { Post-test }(n=48)\end{array}$ & $\begin{array}{c}\text { All } \\
\text { Post-test }(n=160)\end{array}$ \\
\hline Constant rate (total) & $95 \%(106)$ & $92 \%(44)$ & $94 \%(150)$ \\
\hline Straight line or constant slope & $27 \%(30)$ & $17 \%(8)$ & $24 \%(38)$ \\
\hline Interval reasoning & $60 \%(67)$ & $48 \%(23)$ & $56 \%(90)$ \\
\hline Slope and interval & $5 \%(6)$ & $23 \%(11)$ & $11 \%(17)$ \\
\hline Other (e.g., proportional reasoning) & $3 \%(3)$ & $4 \%(2)$ & $3 \%(5)$ \\
\hline Rate increases & $5 \%(6)$ & $0 \%(0)$ & \\
\hline Rate decreases & $0 \%(0)$ & $8 \%(4)$ & \\
\hline Do not know or no answer & $0 \%(0)$ & $0 \%(0)$ & $0 \%(0)$ \\
\hline
\end{tabular}

TABLE X. Post-test results linking the reasons students gave for correctly identifying constant rate with how they determined a value for the rate of change at one particular instant. For an example of how to read the table, see the caption of Table II.

\begin{tabular}{lccccccc}
\hline \hline & \multicolumn{3}{c}{ Interval reasoning only } & & & & \\
$\begin{array}{l}\text { Approaches to correctly identifying } \\
\text { constant rate of change }\end{array}$ & Qualitative & Quantitative & All & Slope only & Slope + interval & Other & Total \\
Approaches to finding & $n=17$ & $n=73$ & $n=90$ & $n=38$ & $n=17$ & $n=5$ & $n=150$ \\
instantaneous rate of change & $(11 \%)$ & $(49 \%)$ & $(60 \%)$ & $(25 \%)$ & $(11 \%)$ & $(3 \%)$ & $(100 \%)$ \\
\hline $\boldsymbol{\Delta} \boldsymbol{y} / \boldsymbol{\Delta} \boldsymbol{x}$ or similar & $\mathbf{5 3 \% ( 9 )}$ & $\mathbf{3 6 \% ( 2 6 )}$ & $\mathbf{3 9 \%}(\mathbf{3 5})$ & $\mathbf{3 7 \%}(\mathbf{1 4 )}$ & $\mathbf{4 7 \% ( 8 )}$ & $\mathbf{4 0 \% ( 2 )}$ & $\mathbf{3 9 \%}(\mathbf{5 9})$ \\
$y / x$ or similar & $35 \%(6)$ & $53 \%(39)$ & $50 \%(45)$ & $50 \%(19)$ & $41 \%(7)$ & $40 \%(2)$ & $49 \%(73)$ \\
Other & $6 \%(1)$ & $8 \%(6)$ & $8 \%(7)$ & $13 \%(5)$ & $0 \%(0)$ & $0 \%(0)$ & $8 \%(12)$ \\
No answer & $6 \%(1)$ & $3 \%(2)$ & $3 \%(3)$ & $0 \%(0)$ & $12 \%(2)$ & $20 \%(1)$ & $4 \%(6)$ \\
\hline \hline
\end{tabular}




\section{E. Conclusions from post-test analysis}

The post-test data allow us to further comment on the research questions.

(1) Difficulties with the direction of motion appear to have disappeared, after a single laboratory-based intervention. The coding categories that emerged from the pretest data still apply. While 20\% of students still struggled with identifying the constancy of speed from a linear distance-time graph, a greater fraction had started to think about it in what we regard a more sophisticated manner, employing interval reasoning. Even so, less than half of our students could successfully determine the speed; this success rate remained independent of the reasoning employed to determine the constancy of the speed.

(2) The greatest improvement was made in the context-free setting, where many more students obtain the correct value for the slope using a correct procedure after instruction. As practically all students could now determine the slope at a point on a $y, x$ graph, it was confirmed that this ability and being able to answer qualitative aspects of numerical distance-time graphs was insufficient to successfully determine a value for the speed from a linear distance-time graph.

(3) Differences between the prevalences of approaches to answering water level versus time graphs and distance-time graphs became smaller after the intervention. The fraction of correct answers obtained, and gains on the type of reasoning used, are similar for the distance-time and water level versus time questions in the post-test. This suggests that the questions were becoming equivalent to a greater fraction of the students (as they would be to experts). However, the overall success rate was at the $40 \%$ level only, the same as the pretest rate for water level versus time graphs.

\section{CONCLUSION}

We have investigated the difficulties first-year university students $(n \approx 550)$ encountered when determining a value for speed from linear distance-time graphs, aided by studies on analogous water level versus time and context-free graphs. The quantitative nature of the questions has allowed us to uncover issues with distance-time graphs that have, to the best of our knowledge, not been reported previously. When answering conceptually equivalent questions in different contexts, students deploy different problem solving strategies. To many students, context seems to be an integral part to the question, even if at a more abstract level the context need not influence the answer.

We have found that neither slope-height confusion nor WYSIWYG effects seem to play an important role. However, we infer from the written answers, semistructured interviews, and classroom interactions that many students do not engage sufficiently with the questions on distance-time graphs. The question of whether or not the speed is constant uses a graphical representation, which cues a particular approach. We infer that postintervention students engaged more with this question, and to the same extent as with equivalent questions on water level versus time graphs. Not only did a greater fraction of students answer the post-test question correctly, some $10 \%$ of students gave more than one reason for their answer.

However, when asked to determine a numerical value for the speed, the wording appears to have cued many students to apply a formula, and they ceased to engage with the graphical representation or the question they had just answered. It appears that in the context of this question, the graph no longer represented information about the nature of the motion, but just served to provide data necessary to find an answer through an equation. Both before and after intervention, this strategy led to answers that are (at least implicitly) inconsistent with the previous answer. However, in students' previous experience in school with linear graphs though the origin, that strategy had been a productive and reliable tool.

We find that the greatest improvement took place on answering questions on slope in the context-free setting. This improvement could have come about without requiring students to change their approach to answering the question; they only needed to replace an incorrect procedure with a better one. Nevertheless, since contextfree graphs were not included in any instruction, and interval reasoning was, it appears that the intervention (and the interval reasoning employed therein) had a positive effect.

Before and after instruction, students answered qualitative questions on water level versus time graphs better than questions on distance-time graphs; differences in answering the numerical question, in terms both of quality and of prevalence, disappeared. This suggests to us that the school experience with the distance-time graphs negatively impacted on the students' problem solving strategy and their likeliness to engage with the question. Their answers to the post-test suggest that a greater fraction of the students engaged with the question than preintervention, but this did not yet result in a much greater fraction being able to answer the question fully correctly. (Indeed, postintervention, some students overrode their earlier correct graphical interpretation when they applied the same incorrect equation to two points on the graph. These students also engaged more deeply with the problem than before, but with an undesired outcome.) The smaller improvement on post-test questions on water level versus time and distancetime graphs compared to context-free settings indicates that technical difficulties played only a small role.

In summary, we have identified and quantified a number of difficulties our students have with numerical linear 
distance-time graphs. The categorization of determining the constancy of speed based on constant slope, interval reasoning, both, or other, appears to be robust and does not change after an intervention, although the prevalences do change. The success rate of determining a value for the speed was found to be low, and independent of the reasoning used to determine the constancy of the speed. It would be interesting to see if these findings may be generalized to other universities in other countries. We have also given some suggestions to explain why such a small fraction of students can successfully determine the speed from a linear distance-time graph. While this aspect of the work is necessarily somewhat speculative in nature, we hope that this may stimulate further work, likely more qualitative in nature, to address these findings.

\section{ACKNOWLEDGMENTS}

We gratefully acknowledge fruitful discussions with David Smith, Paul Swift, Scott McDonald, Odilla Finlayson, Ton Ellermeijer, and Mieke De Cock and assistance in the laboratory from Nicola Alcorn, Mike Aughey, Henry Barry, Des Lavelle, and many postgraduate teaching assistants. This work was supported by the Irish Research Council for Science Engineering and Technology, Dublin City University's Learning Innovation Unit, and the School of Physical Sciences.
[1] T. P. Carpenter, M. K. Corbitt, H. S. Kepner, Jr., M. M. Lindquist, and R.E. Reys, Decimals: Results and implications from the second NAEP mathematics assessment, Arithmetic Teacher 28, 34 (1981) [http://www.jstor.org/ stable/41191870].

[2] G. Leinhardt, O. Zaslavsky, and M. K. Stein, Functions, graphs, and graphing: Tasks, learning, and teaching, Rev. Educ. Res. 60, 1 (1990).

[3] D. Kerslake, Graphs, in Children's Understanding of Mathematics, edited by K.M. Hart (John Murray, London, 1981), pp. 120-136.

[4] L. C. McDermott, M. L. Rosenquist, and E. H. Van Zee, Student difficulties in connecting graphs and physics: Examples from kinematics, Am. J. Phys. 55, 503 (1987).

[5] D. L. McKenzie and M. J. Padilla, The construction and validation of the test of graphing in science (togs), J. Res. Sci. Teach. 23, 571 (1986).

[6] J.R. Mokros and R.F. Tinker, The impact of microcomputer-based labs on children's ability to interpret graphs, J. Res. Sci. Teach. 24, 369 (1987).

[7] J. Clement, The concept of variation and misconceptions in Cartesian graphing, Focus Learn. Probl. Math. 11, 77 (1989).

[8] R. K. Thornton and D. R. Sokoloff, Learning motion concepts using real-time microcomputer-based laboratory tools, Am. J. Phys. 58, 858 (1990).

[9] An extensive list of further references can be found in L. C. McDermott and E.F. Redish, Resource letter: PER-1: Physics education research, Am. J. Phys. 67, 755 (1999).

[10] R. V. Kail and J. C. Cavanaugh, Human Development: A Life-Span View (Wadsworth Publishing, Belmont, CA, 2008), 5th ed.

[11] F. Matsuda, Development of concepts of interrelationships among duration, distance, and speed, Int. J. Behav. Dev. 25, 466 (2001).

[12] The quote is taken from M. O'Callaghan, S. Reilly, and A. Seery, Exploring Science (Edco, Dublin, 2007), p. 250. Almost identical quotes may be found in the most popular textbooks in use in Ireland.
[13] P. W. Thompson, The development of the concept of speed and its relationship to concepts of rate, in The Development of Multiplicative Reasoning in the Learning of Mathematics, edited by G. Harel and J. Confrey (SUNY Press, Albany, NY, 1994), pp. 179-234.

[14] B.L. Sherin, How students invent representations of motion. A genetic account, J. Math. Behav. 19, 399 (2000).

[15] J.P. Smith III, A. DiSessa, and J. Roschelle, Misconceptions reconceived: A constructivist analysis of knowledge in transition, J. Learn. Sci. 3, 115 (1994).

[16] A. A. diSessa, Toward an epistemology of physics, Cognit. Instr. 10, 105 (1993); Between brain and behavior: Response to Marton, Cognit. Instr. 10, 261 (1993).

[17] J. Minstrell, Facets of students' knowledge and relevant instruction, in Proceedings of an International Workshop-Research in Physics Learning: Theoretical Issues and Empirical Studies, edited by R. Duit, F. Goldberg, and H. Niedderer [The Institute for Science Education (IPN), Kiel, Germany, 1982], pp. 110-128.

[18] J. G. Greeno, Gibson's affordances, Psychol. Rev. 101, 336 (1994).

[19] W. M. Roth, Cascades of inscriptions and re-presentation of nature: How numbers, tables, graphs, and money come to re-present a rolling ball, Int. J. Sci. Educ. 19, 1075 (1997).

[20] L. Åberg-Bengtsson and T. Ottosson, What lies behind graphicacy? Relating students' results on a test of graphically represented quantitative information to formal academic achievement, J. Res. Sci. Teach. 43, 43 (2006).

[21] A. F. Heckler, T. M. Scaife, and E. C. Sayre, Response times and misconception-like responses to science questions, in Proceedings of XXXII Cog SCi Conference, 2010 (unpublished), pp. 139-144.

[22] A. Elby, What students' learning of representations tells us about constructivism, J. Math. Behav. 19, 481 (2000).

[23] It is worth emphasizing that, even though some students calculate $y / x$ instead of $\Delta y / \Delta x$, and thus arguably confuse the ratio of two intervals with the ratio of two coordinates of a point, this is not considered interval-point 
confusion. The name interval-point confusion is reserved for the much simpler cases described in Sec. I A, and the questions we asked our students did not probe whether interval-point confusion was prevalent.

[24] R. J. Beichner, Testing student interpretation of kinematics graphs, Am. J. Phys. 62, 750 (1994).

[25] The majority of our students have taken physics only up to age 15 as part of a general Science course. While other distance-time graphs are typically shown in textbooks, they only truly interact with and extract information from linear distance-time graphs through the origin.

[26] For a thorough discussion on turning qualitative analysis into a quantitative study, see M.T.H. Chi, Quantifying qualitative analyses of verbal data: A practical guide, J. Learn. Sci. 6, 271 (1997).

[27] In this context, it is interesting to note that in Ref. [21], Heckler et al. showed that imposing a 3 s delay on students answering questions on qualitative graphs improved the percentage of correct responses from 50 to 70 .

[28] H. Brasell, The effect of real-time laboratory graphing on learning graphic representations of distance and velocity, J. Res. Sci. Teach. 24, 385 (1987).
[29] A. Hofstein and V. N. Lunetta, The laboratory in science education: Foundations for the twenty-first century, Sci. Educ. 88, 28 (2004).

[30] M. J. Wavering, Logical reasoning necessary to make line graphs, J. Res. Sci. Teach. 26, 373 (1989).

[31] C. A. Berg and P. Smith, Assessing students abilities to construct and interpret line graphs: Disparities between multiple-choice and free-response instruments, Sci. Educ. 78, 527 (1994).

[32] P. W. Laws, Millikan lecture 1996: Promoting active learning based on physics education research in introductory physics courses, Am. J. Phys. 65, 14 (1997).

[33] L. C. McDermott (Physics Education Group), Physics by Inquiry (Wiley, New York, 1996), pp. 639-640.

[34] R. R. Hake, Interactive engagement versus traditional methods: A six-thousand student survey of mechanics test data for introductory physics courses, Am. J. Phys. 66, 64 (1998). For a thorough discussion on the validity of $h$, see also D. E. Meltzer, The relationship between mathematics preparation and conceptual learning gains in physics: A possible hidden variable in diagnostic pretest scores, Am. J. Phys. 70, 1259 (2002). 